PROCEEDINGS OF THE

AMERICAN MATHEMATICAL SOCIETY

Volume 128, Number 5, Pages 1497-1506

S 0002-9939(00)05385- 5

Article electronically published on February 3, 2000

\title{
CONNECTED SUMS OF MANIFOLDS WHICH INDUCE APPROXIMATE FIBRATIONS
}

\author{
YONGKUK KIM
}

(Communicated by Ralph Cohen)

\begin{abstract}
Codimension-2 fibrators are $n$-manifolds which automatically induce approximate fibration, in the following sense: given any proper mapping $p$ from an $(n+2)$-manifold onto a 2-manifold such that each point-preimage is a copy of the codimension-2 fibrator, $p$ is necessarily an approximate fibration. In this paper, we give some answers to the following question: given an $n$-manifold $N$ which is a nontrivial connected sum, when is $N$ a codimension-2 fibrator?
\end{abstract}

\section{INTRODUCTION}

There is a useful class of mappings called approximate fibrations; namely, those proper mappings which satisfy an approximate version of the homotopy lifting property defining the more familiar class of fibrations. It is well known that if a proper map is an approximate fibration, then the point inverses are shape equivalent to each other. Naturally, the question arises about conditions under which the converse of this fact holds. To deal with that, in [5] R.J. Daverman introduced the following definition: a closed $n$-manifold $N^{n}$ is a codimension-2 fibrator (respectively, a codimension-2 orientable fibrator) if, whenever $p: M \longrightarrow B$ is a proper map from an arbitrary (respectively, orientable) $(n+2)$-manifold $M$ to a 2-manifold $B$ such that each $p^{-1}(b)$ is shape equivalent to $N$, then $p: M \longrightarrow B$ is an approximate fibration. The main problem is to determine which manifolds $N$ are codimension-2 fibrators. Simply connected manifolds, finite products of closed surfaces with nonzero Euler characteristic, closed manifolds $N$ with $\pi_{1}(N) \cong \mathbb{Z}_{2}$ (for example, real projective $n$-spaces $(n>1)$ ), and closed manifolds with finite (or abelian) fundamental group and nonzero Euler characteristic are known to be codimension-2 fibrators ([1, Theorem 4.1], [5, Corollary 2.4 and Theorem 5.1] and [16, Corollary 3.1]). Also, closed hopfian manifolds with hopfian fundamental group and nonzero Euler characteristic as well as closed hopfian manifolds with hyperhopfian fundamental group are known to be codimension-2 orientable fibrators ( 6 . Corollary 5.5 and Theorem 5.10]).

Surprisingly $R P^{n} \# R P^{n}$ is not a codimension-2 fibrator, while $R P^{n}$ is $\underline{6}$, Theorem 4.2]. But in this paper, we show that most closed hopfian $n$-manifolds

Received by the editors February 12, 1998.

1991 Mathematics Subject Classification. Primary 57N15, 55M25; Secondary 57M10, 54B15.

Key words and phrases. Connected sum, approximate fibration, codimension-2 fibrator, hopfian manifold, hyperhopfian group, residually finite group. 
$N^{n}=N_{1} \# N_{2}$ with hopfian $\pi_{1}\left(N_{1}\right), \pi_{1}\left(N_{2}\right)$ and $H_{1}\left(N_{1}\right) \cong \mathbb{Z}_{2} \cong H_{1}\left(N_{2}\right)$ are codimension- 2 orientable fibrators (see Proposition 3.3 below).

We study the behavior of codimension-2 fibrators under the connected sum operation. In other words, we discuss the following

Question. When is a connected sum of two closed manifolds a codimension-2 fibrator?

First we review the known cases under which connected sums of two closed $n$ manifolds $(n \geq 3)$ are codimension-2 fibrators (see Proposition 3.2 below). Then we consider the major nonsettled case which is a connected sum $N$ of a simply connected manifold $N_{1}$ and a nonsimply connected manifold $N_{2}$. To get the orientationfree version about codimension-2 fibrator, we use a new concept of s-hopfianness which is slightly different from strong hopfianness. We show that if $N$ is s-hopfian and $\pi_{1}\left(N_{2}\right)$ is hopfian, and if the simply connected manifold $N_{1}$ has a nonzero betti number, then $N$ is a codimension-2 fibrator. As a consequence, we will get the neat answer on orientable 4-manifolds as follows: Given a closed orientable 4-manifold $N$ which is a nontrivial connected sum of two 4-manifolds $N_{1}$ and $N_{2}$, if $\pi_{1}\left(N_{1}\right)$ and $\pi_{1}\left(N_{2}\right)$ are hopfian, then $N$ is a codimension-2 fibrator. In closing, we deal with some of the remaining possible situations when $N_{1}$ is a rational homology sphere.

\section{Preliminaries}

Throughout this paper, the symbols $\chi, \sim, \approx$ and $\cong$ denote Euler characteristic, homotopy equivalence, homeomorphism and isomorphism in that order. All manifolds are understood to be finite dimensional, connected, metric, and boundaryless. Whenever the presence of boundary is tolerated, the object will be called a manifold with boundary.

A proper map $p: M \longrightarrow B$ between locally compact $A N R \mathrm{~s}$ is called an $a p$ proximate fibration if it has the following approximate homotopy lifting property: Given an open cover $\epsilon$ of $B$, an arbitary space $X$, and two maps $g: X \longrightarrow M$ and $F: X \times I \longrightarrow B$ such that $p \circ g=F_{0}$, there exists a map $G: X \times I \longrightarrow M$ such that $G_{0}=g$ and $p \circ G$ is $\epsilon$-close to $F$. The latter means that for each $z \in X \times I$ there exists a $U_{z} \in \epsilon$ such that $\{F(z), p \circ G(z)\} \subset U_{z}$.

Let $N^{n}$ be a closed manifold. A proper map $p: M \longrightarrow B$ is $N^{n}$-like if each fiber $p^{-1}(b)$ is shape equivalent to $N$. For simplicity or familiarity, we shall assume each fiber $p^{-1}(b)$ in an $N^{n}$-like map to be an $A N R$ having the homotopy type of $N^{n}$.

Let $N$ and $N^{\prime}$ be closed $n$-manifolds and $f: N \longrightarrow N^{\prime}$ a map. If both $N$ and $N^{\prime}$ are orientable, then the degree of $f$ is the nonnegative integer $d$ such that the induced endomorphism of $f_{*}: H_{n}(N ; \mathbb{Z}) \cong \mathbb{Z} \longrightarrow H_{n}\left(N^{\prime} ; \mathbb{Z}\right) \cong \mathbb{Z}$ amounts to multiplication by $d$, up to sign. In general, $f$ has degree $1(\bmod 2)$ if $f_{*}$ : $H_{n}\left(N ; \mathbb{Z}_{2}\right) \longrightarrow H_{n}\left(N^{\prime} ; \mathbb{Z}_{2}\right)$ is an isomorphism and otherwise it has degree $0(\bmod$ $2)$.

Suppose that $N$ is a closed $n$-manifold and a proper map $p: M \longrightarrow B$ is $N$-like. Let $G$ be the set of all fibers, i.e., $G=\left\{p^{-1}(b): b \in B\right\}$. Put $C=\{p(g) \in B: g \in G$ and there exist a neighborhood $U_{g}$ of $g$ in $M$ and a retraction $R_{g}: U_{g} \longrightarrow g$ such that $R_{g} \mid g^{\prime}: g^{\prime} \longrightarrow g$ is a degree one map for all $g^{\prime} \in G$ with $g^{\prime} \in G$ in $U_{g}$, and $C^{\prime}=\left\{p(g) \in B: g \in G\right.$ and there exist a neighborhood $U_{g}$ of $g$ in $M$ and a retraction $R_{g}: U_{g} \longrightarrow g$ such that $R_{g} \mid g^{\prime}: g^{\prime} \longrightarrow g$ is a degree one mod 2 map for all $g^{\prime} \in G$ with $g^{\prime} \in G$ in $\left.U_{g}\right\}$. Call $C$ the continuity set of $p$ and $C^{\prime}$ the $\bmod 2$ 
continuity set of $p$. D. Coram and P. Duvall [4] showed that $C$ and $C^{\prime}$ are dense, open subsets of $B$.

A group $\Gamma$ is said to be hopfian if every epimorphism $f: \Gamma \longrightarrow \Gamma$ is necessarily an isomorphism. A finitely presented group $\Gamma$ is said to be hyperhopfian if every homomorphism $f: \Gamma \longrightarrow \Gamma$ with $f(\Gamma)$ normal and $\Gamma / f(\Gamma)$ cyclic is an isomorphism (onto). A group $\Gamma$ is said to be residually finite if for any nontrivial element $x$ of $\Gamma$ there is a homomorphism $f$ from $\Gamma$ onto a finite group $K$ such that $f(x) \neq 1_{K}$. It is well known that every finitely generated residually finite group is hopfian. Call a closed manifold $N$ hopfian if it is orientable, and every degree one map $N \longrightarrow N$ which induces a $\pi_{1}$-isomorphism is a homotopy equivalence.

The following is basic for investigating codimension-2 fibrators.

Proposition 2.1 ([5] Proposition 2.8]). Let $p: M \longrightarrow B$ be a map from an orientable $(n+2)$-manifold $M$ onto a metric space $B$ such that each $p^{-1}(b)$ is an orientable n-manifold up to shape. Then $B$ is a 2-manifold and $D=B \backslash C$ is locally finite in $B$, where $C$ represents the continuity set of $p$. Moreover, if either $M$ or some $p^{-1}(b)$ are nonorientable, $B$ is a 2-manifold with boundary (possibly empty) and $D^{\prime}=($ int $B) \backslash C^{\prime}$ is locally finite in $B$, where $C^{\prime}$ represents the mod 2 continuity set of $p$.

The next result summarizes useful information connecting hopfian manifolds and hopfian fundamental groups.

Proposition 2.2 ([6] Theorem 2.2] or [13]). A closed, orientable n-manifold $N$ is a hopfian manifold if any one of the following conditions holds:

1) $n \leq 4$ and $\pi_{1}(N)$ is hopfian;

2) $\pi_{1}(N)$ contains a nilpotent subgroup of finite index;

3) $\pi_{1}(N)$ is hopfian and $\pi_{i}(N)$ is trivial for $1<i<n-1$.

To get some orientation-free version about fibrators, we use the following, which is a technical improvement to the definition of a strongly hopfian manifold (see [16]).

Definition. A closed manifold $N$ is s-hopfian if $N$ is hopfian when $N$ is orientable, and $N_{H}$ is hopfian when $N$ is nonorientable, where $N_{H}$ is the covering space of $N$ corresponding to $H=\bigcap_{i \in I} H_{i}$ with $I=\left\{i:\left[\pi_{1}(N): H_{i}\right]=2\right\}$.

Remark. 1) By M. Hall's Theorem (for any finitely generated group $G$, the number of subgroups of $G$ having any fixed finite index is finite), the index set $I$ is finite, and so $H$ has a finite index in $\pi_{1}(N)$.

2) It follows from Proposition 2.2 that closed manifolds with abelian or finite fundamental group and closed aspherical manifolds with residually finite fundamental group are examples of s-hopfian manifolds.

Proposition 2.3. Let $N$ be an s-hopfian $n$-manifold with hopfian fundamental group. Let a proper map $p: M \rightarrow B$ defined on an $(n+2)$-manifold $M$ be $N$ like. Then, $p$ is an approximate fibration over some dense open subset $O$ of the mod 2 continuity set $C^{\prime}$ of $p$, and $C^{\prime} \backslash O$ is locally finite.

Proof. It follows from the proof of [17. Theorem 3.3] for the nonorientable case and [15, Theorem 3.1] for the orientable case.

Proposition 2.4. Let $N$ be an s-hopfian closed manifold. If $\pi_{1}(N)$ is hopfian and $\chi(N) \neq 0$, or if $\pi_{1}(N)$ is hyperhopfian, then $N$ is a codimension-2 fibrator. 
Proof. It follows from [16. Theorem 3.3] and [17, Theorem 3.3] for the nonorientable case and [15, Corollary 3.1] for the orientable case.

\section{Connected Sums of MANifolds}

Notation. We use $N_{1}^{n} \# N_{2}^{n}$ to denote a connected sum of two $n$-manifolds $N_{1}$ and $N_{2}(n \geq 3)$, where neither $N_{1}$ nor $N_{2}$ is a homotopy sphere. Then $N$ is of the form $K_{1} \cup K_{2}$, where $K_{1} \cap K_{2}=S^{n-1}$ and $K_{1}=N_{1} \backslash\left(\right.$ int $\left.B^{n}\right), K_{2}=N_{2} \backslash\left(\right.$ int $\left.B^{n}\right)$. We reserve the notations $K_{1}$ and $K_{2}$ for this purpose throughout this section.

Let us begin with a technical lemma.

Lemma 3.1. Let $X$ and $Y$ be closed orientable $n$-manifolds and $f: X \rightarrow Y$ a map with nonzero degree $t$. Then,

1) $\beta_{i}(X) \geq \beta_{i}(Y)$ for all $i$.

2) If there exists a $j$ such that $\left(\left|T H_{j}(Y)\right|, t\right)=1$, where $T H_{j}(Y)$ is the torsion subgroup of $H_{j}(Y)$, then $H_{j}(Y)$ is isomorphic to a subgroup of $H_{j}(X)$.

3) If there exists a torsion group $H_{j}(Y)$ such that $\left(\left|H_{j}(Y)\right|, t\right)=1$, then $f^{*}$ : $H^{n-j}(Y) \rightarrow H^{n-j}(X)$ is injective and $f_{*}: H_{j}(X) \rightarrow H_{j}(Y)$ is surjective.

4) For any prime number $s$ with $(s, t)=1, f^{*}: H^{n-j}\left(Y ; \mathbb{Z}_{s}\right) \rightarrow H^{n-j}\left(X ; \mathbb{Z}_{s}\right)$ is injective and $f_{*}: H_{j}\left(X ; \mathbb{Z}_{s}\right) \rightarrow H_{j}\left(Y ; \mathbb{Z}_{s}\right)$ is surjective.

Proof. Let $\Gamma_{X}$ and $\Gamma_{Y}$ be the orientation classes of $X$ and $Y$, respectively. Let $G$ be an abelian group. Define $g: H^{n-i}(X ; G) \rightarrow H^{n-i}(Y ; G)$ by $g=D_{Y}^{-1} \circ f_{*} \circ D_{X}$, where $D_{X}$ and $D_{Y}$ are the Poincaré duality maps. Consider the following commutative diagram:

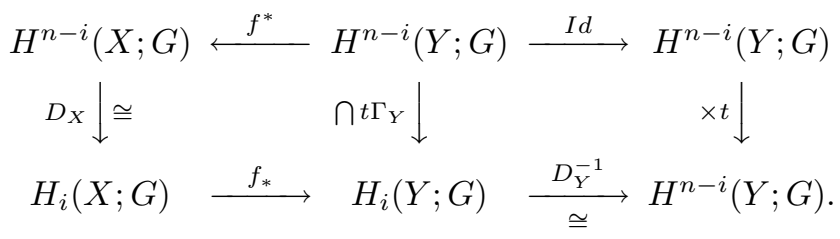

Then, since $g\left(f^{*}(a)\right)=t a, f^{*}$ is monic mod torsion, so we have that $\beta_{i}(Y) \leq \beta_{i}(X)$.

2) and 3) follow immediately from the fact that $\left(g \circ f^{*}\right)=(\times t) \circ I d: H^{n-i}(Y) \rightarrow$ $H^{n-i}(Y)$ is an injection.

4) In the above diagram, since $(s, t)=1$, we see that $\bigcap t \Gamma_{Y}: H^{n-i}\left(Y ; \mathbb{Z}_{s}\right) \rightarrow$ $H_{i}\left(Y ; \mathbb{Z}_{s}\right)$ is an isomorphism. This proves 4$)$.

Proposition 3.2. Let $N=N_{1} \# N_{2}$ be a closed $n$-manifold $(n \geq 3)$. Then $N$ is a codimension-2 fibrator if any one of the following conditions holds:

1) $\pi_{1}\left(N_{1}\right)$ is trivial and $\left|\pi_{1}\left(N_{2}\right)\right| \leq 2$;

2) $N$ is s-hopfian, $\pi_{1}\left(N_{1}\right)$ and $\pi_{1}\left(N_{2}\right)$ are hyperhopfian;

3) $N$ is s-hopfian, $\pi_{1}\left(N_{1}\right)$ and $\pi_{1}\left(N_{2}\right)$ are nontrivial hopfian, and $\pi_{1}(N) \neq$ $\mathbb{Z}_{2} * \mathbb{Z}_{2}$.

Proof. First, we see that $\pi_{1}(N) \cong \pi_{1}\left(N_{1}\right) * \pi_{1}\left(N_{2}\right)$ by the van Kampen Theorem so that $\pi_{1}(N)$ is hopfian, because the free product of two finitely generated hopfian groups is again hopfian [11] Theorem 1.1]. 1) follows from [5, Corollary 2.4 or Theorem 5.2]. Under either condition 2) or 3), $\pi_{1}(N)$ is hyperhopfian ([6, Corollary 4.12 or Corollary 4.13]) so that by Proposition 2.4, $N$ is a codimension-2 fibrator. 
Proposition 3.2 yields that a closed orientable 3 -manifold $N^{3}=N_{1} \# N_{2}$, where $\pi_{1}\left(N_{1}\right)$ and $\pi_{1}\left(N_{2}\right)$ are hopfian, is a codimension-2 orientable fibrator except when $\pi_{1}(N)=\mathbb{Z}_{2} * \mathbb{Z}_{2}$. Since $R P^{n} \# R P^{n}$ is a non-codimension-2 fibrator, we have such an exception. However, the next proposition says that most closed hopfian $n$-manifolds $N^{n}=N_{1} \# N_{2}$, where $\pi_{1}\left(N_{1}\right) \cong \mathbb{Z}_{2}$ and $\pi_{1}\left(N_{2}\right) \cong \mathbb{Z}_{2}$ are codimension-2 orientable fibrators.

Proposition 3.3. Let $N=N_{1} \# N_{2}$ be a closed hopfian $n$-manifold $(n \geq 3)$ with hopfian $\pi_{1}\left(N_{i}\right)$ and $H_{1}\left(N_{i}\right) \cong \mathbb{Z}_{2}$ for $i=1,2$. Then $N$ is a codimension-2 orientable fibrator if any one of the following conditions holds:

1) there are prime numbers $s, t>2$ (not necessarily distinct) such that $H_{j}\left(N_{1} ; \mathbb{Z}_{s}\right)$ $\neq 0$ and $H_{k}\left(N_{2} ; \mathbb{Z}_{t}\right) \neq 0$ for some indices $j, k$ with $0<j, k<n$.

2) $\left|H_{j}\left(N_{1} ; \mathbb{Z}_{2}\right)\right|>\left|H_{j}\left(N_{2} ; \mathbb{Z}_{2}\right)\right|$ and $\left|H_{k}\left(N_{2} ; \mathbb{Z}_{2}\right)\right|>\left|H_{k}\left(N_{1} ; \mathbb{Z}_{2}\right)\right|$ for some indices $j, k$ with $0<j, k<n$.

Proof. Let $p: M^{n+2} \rightarrow B^{2}$, defined on an orientable $(n+2)$-manifold $M$, be an $N$ like proper map. Since $N$ is hopfian and the free product of two finitely generated hopfian groups is again hopfian [11, Theorem 1.1], $p$ is an approximate fibration over the continuity set $C$ of $p$ [6, Theorem 2.1]. Now, in light of Proposition 2.1, we can localize to the situation in which $B=E^{2}$ and $p$ is an approximate fibration over $C=E^{2} \backslash 0$. Use $g_{0}$ to represent $p^{-1}(0)$, which is a strong deformation retract of $M$ under a retraction $R: M \rightarrow g_{0}$. Put $G=\left\{p^{-1}(b): b \in B\right\}$. Denote $R \mid g$ by $R_{g}$ for $g \in G$. Assume that $\left(R_{g}\right)_{\#}\left(\pi_{1}(g)\right) \neq \pi_{1}\left(g_{0}\right)$. Take the covering $q: g_{0}^{*} \rightarrow g_{0}$ corresponding to $\left(R_{g}\right)_{\#}\left(\pi_{1}(g)\right)$. Then there is a lifting $\overline{R_{g}}: g \rightarrow g_{0}^{*}$ of $R_{g}$ so that $q \circ \overline{R_{g}}=R_{g}$. Since $(\operatorname{deg} q)\left(\operatorname{deg} \overline{R_{g}}\right)=\operatorname{deg} R_{g}=2$ and $\operatorname{deg} q=2$ (see [9] for the proof), deg $\overline{R_{g}}=1$ so that $\overline{\left(R_{g}\right)_{*}}: H_{1}(g) \rightarrow H_{1}\left(g_{0}^{*}\right)$ is onto. Hence $H_{1}\left(g_{0}^{*}\right)$ should be finite. But since $g_{0}^{*}$ has the homotopy type of $N^{*}$, where $N^{*}$ is a 2-to- 1 covering space of $N, g_{0}^{*}$ is formed either by two copies of $K_{1}$ and $q^{-1}\left(K_{2}\right)$ or by $q^{-1}\left(K_{1}\right)$ and two copies of $K_{2}$ or by a 2-to- 1 covering space $L$ of $N$ with $H_{1}(L)$ containing a $\mathbb{Z}$ factor. Since $H_{1}\left(g_{0}^{*}\right)$ is finite, the third case cannot happen. Without loss of generality, we assume that $g_{0}^{*}$ is of the first form.

Proof of 1). By Lemma 3.1, we see that $\left(R_{g}\right)_{*},{\overline{\left(R_{g}\right)_{*}}}_{\text {and }} q_{*}$ are surjective on $\mathbb{Z}_{s}$-homology. Consider the following commutative diagram:

$$
\begin{aligned}
& H_{j}\left(g ; \mathbb{Z}_{s}\right) \stackrel{{\overline{\left(R_{g}\right)_{*}}}_{\text {onto }}}{\stackrel{\text { no }}{\longrightarrow}} H_{j}\left(g_{0}^{*} ; \mathbb{Z}_{s}\right) \\
& I d \downarrow \quad q_{*} \downarrow \text { onto } \\
& H_{j}\left(g ; \mathbb{Z}_{s}\right) \stackrel{\left(R_{g}\right)_{*}}{\underset{\text { onto }}{\longrightarrow}} H_{j}\left(g_{0} ; \mathbb{Z}_{s}\right) \text {. }
\end{aligned}
$$

Since $H_{j}\left(g ; \mathbb{Z}_{s}\right) \cong H_{j}\left(g_{0} ; \mathbb{Z}_{s}\right)$ is hopfian, $\left(R_{g}\right)_{*}$ is an isomorphism, so $\overline{\left(R_{g}\right)_{*}}$ : $H_{j}\left(g_{0} ; \mathbb{Z}_{s}\right) \rightarrow H_{j}\left(g_{0}^{*} ; \mathbb{Z}_{s}\right)$ is an isomorphism.

On the other hand, by Lemma 3.1 again, we have that $q^{*}: H^{n-j}\left(g_{0} ; \mathbb{Z}_{s}\right) \rightarrow$ $H^{n-j}\left(g_{0}^{*} ; \mathbb{Z}_{s}\right)$ and $q^{*}: H^{n-j}\left(K_{2} ; \mathbb{Z}_{s}\right) \rightarrow H^{n-j}\left(q^{-1}\left(K_{2}\right) ; \mathbb{Z}_{s}\right)$ are injective so that $H_{j}\left(K_{2} ; \mathbb{Z}_{s}\right)$ and $H_{j}\left(g_{0} ; \mathbb{Z}_{s}\right)$ are isomorphic to subgroups of $H_{j}\left(q^{-1}\left(K_{2}\right) ; \mathbb{Z}_{s}\right)$ and $H_{j}\left(g_{0}^{*} ; \mathbb{Z}_{s}\right)$, respectively. Since $H_{j}\left(K_{1} ; \mathbb{Z}_{s}\right)$ is nontrivial, $\left|H_{j}\left(g_{0} ; \mathbb{Z}_{s}\right)\right|<\left|H_{j}\left(g_{0}^{*} ; \mathbb{Z}_{s}\right)\right|$, which gives a contradiction. 
Proof of 2). Since $\operatorname{deg} \overline{R_{g}}=1$, we have an epimorphism $\overline{\left(R_{g}\right)_{*}}: H_{1}\left(g ; \mathbb{Z}_{2}\right) \rightarrow$ $H_{1}\left(g_{0}^{*} ; \mathbb{Z}_{2}\right)$. But since $\left|H_{j}\left(N_{1} ; \mathbb{Z}_{2}\right)\right|>\left|H_{j}\left(N_{2} ; \mathbb{Z}_{2}\right)\right|$, as before we see that $\left|H_{j}\left(g_{0} ; \mathbb{Z}_{2}\right)\right| \leq\left|H_{j}\left(g_{0}^{*} ; \mathbb{Z}_{2}\right)\right|$, which gives a contradiction.

Proposition 3.2 makes us consider the remaining nonsettled case: given a closed $n$-manifold $N=N_{1} \# N_{2}(n \geq 4)$, what if $\pi_{1}\left(N_{1}\right)$ is trivial and $\pi_{1}\left(N_{2}\right)$ is not?

Proposition 3.4. Let $N=N_{1} \# N_{2}$ be an s-hopfian closed $n$-manifold $(n \geq 4)$ with trivial $\pi_{1}\left(N_{1}\right)$ and hyperhopfian $\pi_{1}\left(N_{2}\right)$. Then $N$ is a codimension-2 fibrator.

Proof. It follows immediately from Proposition 2.4.

Proposition 3.5. Let $N=N_{1} \# N_{2}$ be an s-hopfian closed $n$-manifold $(n \geq 4)$ with trivial $\pi_{1}\left(N_{1}\right)$ and hopfian $\pi_{1}\left(N_{2}\right)$. If $\beta_{j}\left(N_{1}\right) \neq 0$ for some $j(0<j<n)$, then $N$ is a codimension-2 fibrator.

Proof. Note that

1) By the van Kampen Theorem, we see that $\pi_{1}(N) \cong \pi_{1}\left(K_{2}\right) \cong \pi_{1}\left(N_{2}\right)$, which is hopfian.

2 ) Using the Mayer-Vietoris sequence, we see that $\beta_{j}\left(K_{1}\right)=\beta_{j}\left(N_{1}\right) \neq 0$. Let $p: M \longrightarrow B$ be an $N$-like proper map defined on an $(n+2)$-manifold $M$. Put $G=\left\{p^{-1}(b): b \in B\right\}$.

\section{Case $\mathbf{I}: N$ is orientable.}

Subcase I: $M$ is orientable.

Since $p$ is an approximate fibration over the continuity set $C$ of $p(\underline{6}$, Theorem $2.1]$ ), in light of Proposition 2.1, we can localize to the situation in which $B=E^{2}$ and $p$ is an approximate fibration over $C=E^{2} \backslash 0$. Use $g_{0}$ to represent $p^{-1}(0)$, which is a strong deformation retract of $M$ under a retraction $R: M \rightarrow g_{0}$. By [6] Lemma 5.3], it suffices to show that the restriction of $R$ induces an epimorphism $\pi_{1}(g) \rightarrow \pi_{1}\left(g_{0}\right)$ for $g \neq g_{0}$.

Take the covering $q: M^{*} \rightarrow M$ corresponding to $i_{\#}\left(\pi_{1}(g)\right)$, where $i: g \rightarrow M$ is the inclusion. Denote the restriction map $R \mid g$ by $R_{g}$.

Subcase 1: $\left[\pi_{1}(M): i_{\#}\left(\pi_{1}(g)\right)\right]=t<\infty$.

Suppose that $t \neq 1$. Then consider

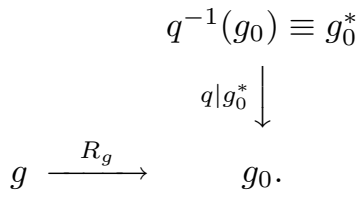

Since $g_{0}^{*}$ is connected and $\pi_{1}\left(g_{0}^{*}\right) \cong\left(R_{g}\right)_{\#}\left(\pi_{1}(g)\right) \cong i_{\#}\left(\pi_{1}(g)\right)$, we have a lifting $\overline{R_{g}}$ of $R_{g}$ so that $\left(q \mid g_{0}^{*}\right) \circ \overline{R_{g}}=R_{g}$. By [6. Lemma 5.2'], $R_{g}$ has positive degree, and so $\overline{R_{g}}$ has positive degree. By Lemma 3.1 , we have $\beta_{j}(g) \geq \beta_{j}\left(g_{0}^{*}\right)$. We also see that $\beta_{j}\left(g_{0}^{*}\right) \geq \beta_{j}\left(g_{0}\right)$ (from [12, Corollary 1]). Since $\beta_{j}(g)=\beta_{j}\left(g_{0}\right)$, we have $\beta_{j}\left(g_{0}^{*}\right)=\beta_{j}\left(g_{0}\right)$.

Now, identify $g_{0}$ with $K_{1} \cup K_{2}$. Put $\left(q \mid g_{0}\right)^{-1}\left(K_{1}\right)_{C}=\left(L_{1}\right)_{C},\left(q \mid g_{0}\right)^{-1}\left(K_{2}\right)=$ $L_{2}$, where $\left(q \mid g_{0}\right)^{-1}\left(K_{1}\right)_{C}$ is a component of $\left(q \mid g_{0}\right)^{-1}\left(K_{1}\right)$. Then, since $(q \mid)$ : $L_{2} \rightarrow K_{2}$ is a finite covering, we have $\beta_{j}\left(L_{2}\right) \geq \beta_{j}\left(K_{2}\right)$ (from [12, Corollary 1]). But since $t>1$, we have $\beta_{j}\left(g_{0}^{*}\right)=t \beta_{j}\left(\left(L_{1}\right)_{C}\right)+\beta_{j}\left(L_{2}\right)=t \beta_{j}\left(K_{1}\right)+\beta_{j}\left(L_{2}\right)>$ $\beta_{j}\left(K_{1}\right)+\beta_{j}\left(K_{2}\right)=\beta_{j}\left(g_{0}\right)$, which gives a contradiction. 
Subcase 2: $\left[\pi_{1}(M): i_{\#}\left(\pi_{1}(g)\right)\right]=\infty$.

If $\left(R_{g}\right)_{\#}: \pi_{1}(g) \rightarrow \pi_{1}\left(g_{0}\right)$ is not an epimorphism, then by the argument in the proof of [6. Theorem 5.10], we have that $q^{-1}\left(g_{0}\right)$ has finitely generated homology, which is impossible because $q^{-1}\left(g_{0}\right)$ contains infinitely many copies of $K_{1}$ and $\beta_{j}\left(K_{1}\right) \neq 0$.

Subcase II: $M$ is nonorientable.

If $N$ has no 2-to-1 covering, by [1, Corollary 3.3] and Subcase $1, N$ is a codimension-2 fibrator. Now assume that $N$ has a 2 -to-1 covering.

Claim: $p$ is an approximate fibration over the mod 2 continuity set $C^{\prime}$ of $p$.

In light of Proposition 2.3, we can localize the situation so that $C^{\prime}$ is an open disk containing $b_{0}=p\left(g_{0}\right)$ and $p$ is an approximate fibration over $C^{\prime} \backslash b_{0}$. Also we may assume that $R: p^{-1}\left(C^{\prime}\right) \longrightarrow g_{0}$ is a strong deformation retraction. Take the covering $q: M^{*} \rightarrow p^{-1}\left(C^{\prime}\right)$ corresponding to $R_{\#}^{-1}(H)=H$, where $H=\bigcap_{i \in I} H_{i}$ with $I=\left\{i:\left[\pi_{1}(N): H_{i}\right]=2\right\}$. Then for $g \in G$ with $p(g) \in C^{\prime}, q^{-1}(g) \sim N_{H}$, where $N_{H}$ is the covering of $N$ corresponding to $H$ (see [17, Theorem 3.3] for the detailed proof). Put $G^{*}=\left\{q^{-1}(g): g \in G\right.$ with $\left.p(g) \in C^{\prime}\right\}$ and let $p^{*}: M^{*} \rightarrow$ $M^{*} / G^{*}$ be the decomposition map. Here note $M^{*} / G^{*}=C^{\prime}$. By the same reasoning as in Subcase I, we see that the mod 2 continuity set $C^{\prime}$ of $p$ equals the continuity set $C\left(p^{*}\right)$ of $p^{*}$. Take any $x \in C^{\prime}$ and carefully take a neighborhood $W_{x} \subset C^{\prime}$ of $x$ such that $R_{x}: p^{-1}\left(W_{x}\right) \rightarrow g_{x} \equiv p^{-1}(x)$ is a strong deformation retraction. For $g \in G$ with $p(g) \in W_{x}$, let $\overline{\left(R_{x} \mid\right)}: q^{-1}(g) \rightarrow q^{-1}\left(g_{x}\right)$ be a map such that $(q \mid) \circ \overline{\left(R_{x} \mid\right)}=\left(R_{x} \mid\right) \circ(q \mid)$. Then the degree of $\left(R_{x} \mid\right)=$ the degree of $\overline{\left(R_{x} \mid\right)}=1$ so that $W_{x}$ is contained in the continuity set $C(p)$ of $p$. But since $N$ is hopfian, $p$ is an approximate fibration over $W_{x}$. It follows from the movability argument 3 . Corollary 3.4] that $p$ is an approximate fibration over $C^{\prime}$.

Claim: $p$ is an approximate fibration over (int $B$ ) and $\partial B=\emptyset$.

Again we localize that $\operatorname{int} B$ is an open disk containing $b_{0}=p\left(g_{0}\right)$ and $p$ is an approximate fibration over int $B \backslash b_{0}$. Also assume that $R:$ int $B \rightarrow g_{0}$ is a strong deformation retraction. Take the covering $q: M^{*} \rightarrow p^{-1}$ (intB) corresponding to $R_{\#}^{-1}(H)=H$, where $H=\bigcap_{i \in I} H_{i}$ with $I=\left\{i:\left[\pi_{1}(N): H_{i}\right]=2\right\}$. For $g\left(\neq g_{0}\right) \in G$, let $i: g \longrightarrow p^{-1}\left(C^{\prime}\right)$ be the inclusion map. Set $K=\left(q \mid g_{C}^{*}\right)_{\#}\left(\pi_{1}\left(g_{C}^{*}\right)\right)$ and $\bar{H}=i_{\#}^{-1}(H)$, where $g_{C}^{*}$ is a component of $q^{-1}(g)$. The argument similar to [16, Theorem 3.3] gives the following facts:

1) $H \subset \bar{H}=K$

2) we only need check the case of $H \neq \bar{H}$;

$3)$ in case $H \neq \bar{H}$, we have $\beta_{i}\left(N_{\bar{H}}\right)=\beta_{i}\left(g_{C}^{*}\right)=\beta_{i}\left(g_{0}^{*}\right)=\beta_{i}\left(N_{H}\right)$ for all $i$, where $\bar{q}: N_{\bar{H}} \rightarrow N$ and $\widetilde{q}: N_{H} \rightarrow N$ are the covering maps corresponding to $\bar{H}$ and $H$, respectively. But since $\beta_{j}\left((\widetilde{q})^{-1}\left(K_{2}\right)\right) \geq \beta_{j}\left((\bar{q})^{-1}\left(K_{2}\right)\right)$, we see that $\beta_{j}\left(N_{H}\right)=$ $\left[\pi_{1}(N): H\right] \beta_{j}\left(K_{1}\right)+\beta_{j}\left((\widetilde{q})^{-1}\left(K_{2}\right)\right)>\left[\pi_{1}(N): \bar{H}\right] \beta_{j}\left(K_{1}\right)+\beta_{j}\left((\bar{q})^{-1}\left(K_{2}\right)\right)=$ $\beta_{j}\left(N_{\bar{H}}\right)$, which gives a contradiction.

Now we will show that $\partial B=\emptyset$. Suppose $\partial B \neq \emptyset$. By [10, 2$]$ there exist $a_{0} \in \partial B$, a neighborhood $U$ of $a_{0}$ in $B$, and a deformation retraction $R: p^{-1}(U) \longrightarrow p^{-1}\left(a_{0}\right)$ such that

(1) $U \approx$ the upper half plane $\left\{(x, y) \in E^{2} \mid y \geq 0\right\}$,

(2) $A=(\partial B) \bigcap U$ is an open arc, and

(3) for all $a \in A, R \mid p^{-1}(a): p^{-1}(a) \longrightarrow p^{-1}\left(a_{0}\right)$ is a homotopy equivalence.

Take the covering $q: M^{*} \rightarrow p^{-1}(U)$ corresponding to $R_{\#}^{-1}(H)$, where $H=$ $\bigcap_{i \in I} H_{i}$ with $I=\left\{i:\left[\pi_{1}(N): H_{i}\right]=2\right\}$. For $g \in G$ with $p(g)=b$, let $i: g \longrightarrow$ 
$p^{-1}(U)$ be the inclusion map. Set $\bar{H}=i_{\#}^{-1}(H)$ and $K=\left(q \mid g_{C}^{*}\right)\left(\pi_{1}\left(g_{C}^{*}\right)\right)$. Take the covering map $\bar{q}: N_{\bar{H}} \rightarrow N$ corresponding to $\bar{H}$. Then, since $g^{*}$ has two (or more) components, we have a $d-1$ covering map $N_{H} \longrightarrow N_{\bar{H}} \approx N_{K}$ with $d>1$ and also have a degree one map $N_{K} \sim q^{-1}\left(p^{-1}(b)\right)_{C} \longrightarrow q^{-1}\left(p^{-1}(a)\right) \sim N_{H}$ (see [16] Lemma 3.2] for more detail), where $\widetilde{q}: N_{H} \rightarrow N$ is the covering map corresponding to $H$. By Lemma 3.1 , we have that $\beta_{j}\left(N_{H}\right) \geq \beta_{j}\left(N_{K}\right)=\beta_{j}\left(N_{\bar{H}}\right) \geq \beta_{j}\left(N_{H}\right)$, i.e., $\beta_{j}\left(N_{H}\right)=\beta_{j}\left(N_{\bar{H}}\right)$. But as before we see that $\beta_{j}\left(N_{H}\right)>\beta_{j}\left(N_{\bar{H}}\right)$, which gives a contradiction.

Case II: $N$ is nonorientable.

Then $M$ must be nonorientable, since there is no usc decomposition of an orientable $(n+2)$-manifold consisting entirely of nonorientable $n$-manifolds [5] Proposition 2.9].

Claim: $p$ is an approximate fibration over the mod 2 continuity set $C^{\prime}$ of $p$.

With the same setting of Subcase II in Case I, since $N_{H}$ is hopfian, $p^{*}$ is an approximate fibration over $W_{x}$ so that $p$ is.

Claim: $p$ is an approximate fibration over (int $B)$ and $\partial B=\emptyset$.

The proof is just a copy of Subcase II in Case I.

Corollary 3.1. Let $N=N_{1} \# N_{2}$ be a closed $n$-manifold $(n \geq 4)$ with trivial $\pi_{1}\left(N_{1}\right)$ and finite or abelian $\pi_{1}\left(N_{2}\right)$. If $\beta_{j}\left(N_{1}\right) \neq 0$ for some $j(0<j<n)$, then $N$ is a codimension-2 fibrator.

Corollary 3.2. Let $N=N_{1} \# N_{2}$ be an s-hopfian closed $n$-manifold $(n \geq 4)$ with trivial $\pi_{1}\left(N_{1}\right)$ and hopfian $\pi_{1}\left(N_{2}\right)$. If $\chi\left(N_{2}\right) \neq 0$, then $N$ is a codimension-2 fibrator.

Proof. If $\beta_{j}\left(N_{1}\right) \neq 0$ for some $j(0<j<n)$, we are done. So assume that $\beta_{j}\left(N_{1}\right)=0$ for all $j(0<j<n)$. Then since $n$ must be even, $\chi\left(N_{1}\right)=2$, so we have that $\chi(N)=\chi\left(K_{1}\right)+\chi\left(K_{2}\right)-\chi\left(S^{n-1}\right)=\chi\left(N_{1}\right)-1+\chi\left(N_{2}\right)-1-0=$ $2-1+\chi\left(N_{2}\right)-1-0=\chi\left(N_{2}\right) \neq 0$. Consequently, we have that $N$ is an $n$-manifold with hopfian $\pi_{1}(N)$ and $\chi(N) \neq 0$. By Proposition 2.4, $N$ is a codimension-2 fibrator.

All previous results give us a neat answer to the question concerning connected sums and codimension-2 fibrators in orientable 4-manifolds.

Theorem 3.6. Let $N=N_{1} \# N_{2}$ be a closed orientable 4-manifold. If $\pi_{1}\left(N_{1}\right)$ and $\pi_{1}\left(N_{2}\right)$ are hopfian, then $N$ is a codimension-2 fibrator.

Proof. Since $\pi_{1}(N)$ is hopfian (see proof of Proposition 3.2), then by Proposition $2.2, N$ is hopfian.

Case I: $\pi_{1}\left(N_{1}\right)$ and $\pi_{1}\left(N_{2}\right)$ are trivial.

Every simply connected manifold is a codimension-2 fibrator [5, Corollary 2.4].

Case II: $\pi_{1}\left(N_{1}\right)$ is trivial and $\pi_{1}\left(N_{2}\right)$ is nontrivial.

Then $H_{2}\left(N_{1}\right)$ must be a free abelian group so that $\beta_{2}\left(N_{1}\right) \neq 0$, otherwise $N_{1}$ is the homotopy sphere.

Case III: $\pi_{1}\left(N_{1}\right)$ and $\pi_{1}\left(N_{2}\right)$ are nontrivial.

Subcase $I: H_{1}\left(N_{1}\right)$ and $H_{1}\left(N_{2}\right)$ are finite.

Then, $\chi(N)=2+\beta_{2}(N) \neq 0$. By Proposition 2.4, $N$ is a codimension-2 fibrator. Subcase II: $H_{1}\left(N_{1}\right)$ or $H_{1}\left(N_{2}\right)$ is nonfinite.

Hyperhopfianness of $\pi_{1}(N)$ comes from Proposition 3.2. Then apply Proposition 2.4 . 
At this point one may ask: What if $\beta_{i}\left(N_{1}\right)=0$ for $0<i<n$ ? In other words, given a closed n-manifold $N=N_{1} \# N_{2}$ with simply connected $N_{1}$, for $0<i<$ $n, \beta_{i}\left(N_{1}\right)=0$, when is $N$ a codimension-2 (orientable) fibrator?

The argument of the proof in Proposition 3.3 gives us a partial answer to that question:

Proposition 3.7. Let $N=N_{1} \# N_{2}$ be a closed hopfian $n$-manifold $(n \geq 3)$ with trivial $\pi_{1}\left(N_{1}\right)$ and hopfian $\pi_{1}\left(N_{2}\right)$. Suppose that for all $i(0<i<n), \beta_{i}\left(N_{1}\right)=0$ and $H_{1}\left(N_{2}\right)$ is finite. If there is a prime number e which divides $\left|H_{j}\left(N_{1}\right)\right|$ for some $j(0<j<n)$ but does not divide $\left|H_{1}\left(N_{2}\right)\right|$, then $N$ is a codimension-2 orientable fibrator. Moreover, if $e=2$, then $N$ is a codimension-2 fibrator.

Note. The arguments we used in this section are not enough to give a complete answer to the following questions:

1) Given a closed $n$-manifold $N=N_{1} \# N_{2}(n \geq 5)$, if $N_{1}$ is simply connected and $\mathrm{N}_{2}$ is nonsimply connected with infinite hopfian $H_{1}\left(N_{2}\right)$, is $N$ a codimension-2 (orientable) fibrator?

2) Given a closed $n$-manifold $N=N_{1} \# N_{2}(n \geq 5)$, if $H_{i}\left(N_{1}\right)$ and $H_{i}\left(N_{2}\right)$ are 2-groups or trivial, is $N$ a codimension-2 (orientable) fibrator?

\section{ACKNOWLEDGMENT}

It is with great pleasure that I thank my thesis advisor, Robert J. Daverman, for his support and guidance during this project.

\section{REFERENCES}

[1] N. Chinen, Manifolds with nonzero Euler characteristic and codimension-2 fibrators, Topology Appl. 86 (1998) 151-167 MR 99c:57052

[2] D.S. Coram and P.F. Duvall, Approximate fibrations, Rocky Mountain J. Math. 7 (1977) 275-288 MR 56:1296

[3] D.S. Coram and P.F. Duvall, Approximate fibrations and a movability condition for maps, Pacific J. Math. 72 ( 1977) 41-56 MR 57:7597.

[4] D.S. Coram and P.F. Duvall, Mappings from $S^{3}$ to $S^{2}$ whose point inverses have the shape of a circle, General Topology Appl. 10 (1979) 239-246 MR 81b:57009

[5] R.J. Daverman, Submanifold decompositions that induce approximate fibrations, Topology Appl. 33 (1989) 173-184 MR 91d:57013

[6] R.J. Daverman, Hyperhopfian groups and approximate fibrations, Compositio Math. 86 (1993) 159-176 MR 94b:55022

[7] R.J. Daverman, Codimension-2 fibrators with finite fundamental groups, Proc. Amer. Math. Soc. 127 (1999) 881-888 CMP 99:06

[8] R.J. Daverman, 3-manifolds with geometric structure and approximate fibrations, Indiana Univ. Math. J. 40 (1991) 1451-1469 MR 92m:55016

[9] R.J. Daverman, Manifolds with finite first homology as codimension 2 fibrators, Proc. Amer. Math. Soc. 113 (1991) 471-477 MR 92a:55015

[10] R.J. Daverman and L.S. Husch, Decompositions and approximate fibrations, Michigan Math. J. 31 (1984) 197-214 MR 86a:54009

[11] I.M.S. Dey and H. Neumann, The Hopf property of free products, Math. Z. 117 (1970) 325-339 MR 43:2099

[12] B. Eckmann, Covering and Betti numbers, Bull. Amer. Math. Soc. 55 (1949) 95-101 MR 10:559a

[13] J.C. Hausmann, Geometric hopfian and nonhopfian situations, Lecture Notes in Pure Appl. Math. Marcel Decker, Inc., NY (1987) 157-166 MR 88f:57031

[14] Y. H. Im, Products of surfaces that induce approximate fibrations, Houston J. Math. 21 no. 2 (1995) 339-348 MR 96c:57038 
[15] Y. H. Im and Y. Kim, Hopfian and strongly hopfian manifolds, Fund. Math. 159 (1999), no. 2 127-134. MR 99j:57023

[16] Y. Kim, Strongly hopfian manifolds as codimension-2 fibrators, Topology Appl. 92 (1999), no. 3 237-245. CMP 99:09

[17] Y. Kim, Manifolds with hyperhopfian fundamental group as codimension-2 fibrators, Topology Appl. To appear

[18] J. Milnor, Infinite cyclic coverings, in Conference on the Topology of Manifolds (J.G. Hocking ed.), Prindle Weber \& Schmidt, Inc., Boston, 1968, 115-133 MR 39:3497

[19] J.R. Munkres, Elements of Algebraic Topology, Addison Wesley Publ. Co., NY, 1984 MR 85m:55001

[20] J. Roitberg, Residually finite, hopfian and co-hopfian spaces, Contemporary mathematics $\mathbf{3 7}$ (1985) 131-144 MR 86i:55009

[21] J. J. Rotman, An Introduction to the Theory of Groups, Springer-Verlag, 1995. MR 95m:20001

Department of Mathematics, The University of Tennessee at Knoxville, Knoxville, TENNESSEE 37996-1300

Current address: Department of Mathematics, Kyungpook National University, Taegu, 702701, Korea

E-mail address: yongkuk@kyungpook.ac.kr 